\title{
Mercado, Consumo
}

\section{Y PATRIMONIALIZACIÓN CULTURAL}

\author{
MARgarita CHAVES \\ ICANH \\ mchaves@icanh.gov.co \\ Mauricio Montenegro \\ UNIVERSIDAD CENTRAL \\ mmontenegror@ucentral.edu.co \\ MARTA ZAMBRANo \\ UNIVERSIDAD NACIONAL \\ lmzambranoe@unal.edu.co
}

\section{Presentación}

\begin{abstract}
demás de presentar e invitar a la lectura del dossier inicial de este número de la Revista Colombiana de Antropología, leste artículo propone un marco analítico y cinco ejes conceptuales para ahondar en el examen de las implicaciones de los procesos de sanción patrimonial en la ampliación del consumo cultural. ${ }^{1}$ En conjunto, los artículos que componen este dossier hacen parte de una iniciativa de investigación ${ }^{2}$ que desde el Instituto Colombiano de Antropología e Historia, Icanh, busca examinar en diversos escenarios la lógica de las intervenciones de productores, agentes y gestores en la patrimonialización y en el mercado de bienes culturales, y evaluar los usos contemporáneos

I. Todos estos textos fueron presentados inicialmente como ponencias en un conversatorio que con el mismo título del dossier y de este artículo fue realizado por el Grupo de Antropología Social del Icanh en marzo de 2009. Los textos han sido ampliados, revisados y evaluados para su publicación.

2. El conjunto del dossier, así como este artículo introductorio, son un avance del proyecto "Mercado, consumo y patrimonialización. Agentes sociales y expansión de las industrias culturales en Colombia". Icanh-Colciencias, No. 743-2009.
\end{abstract} de la noción de patrimonio inmaterial, las negociaciones de sentido y los conflictos de poder que este concepto plantea. Así, antes que una revisión exhaustiva de los presupuestos conceptuales y empíricos que orientan los artículos, este texto es parte y resultado parcial del proyecto 
de investigación mencionado. Reflexiona sobre los procesos y agenciamientos involucrados en los procesos de patrimonialización y examina sus relaciones con el mercado. A la vez formula una invitación abierta a nuevas propuestas que contribuyan a la consolidación de un programa de investigación sobre los temas mencionados. Llama también a la participación más activa del campo académico en la re-orientación de las políticas públicas sobre procesos culturales y sociales.

Para empezar, hay que poner de presente que, siguiendo una tendencia global que recoge las recomendaciones de las convenciones de la Unesco, en especial la de protección del patrimonio inmaterial de 2003, el Ministerio de Cultura ha puesto en marcha una serie de normativas que promueven la valoración y salvaguardia de expresiones, saberes y bienes populares y étnicos definidos como de naturaleza inmaterial (Ministerio de Cultura, 2009b). La ley II85 de 2008, el decreto 294I de 2009 y la resolución 0330, en particular, reglamentan los procesos de inventario, registro, gestión y preservación, extendiendo valores y métodos museológicos a los conocimientos, las prácticas, los artefactos, los mundos sociales y los espacios de grupos y sociedades en las que predomina la trasmisión de la tradición por vía oral (Kirshenblatt-Gimblett, B., 2007).

Este proceso, como lo señala B. Kirshenblatt-Gimblett (2004), encierra una paradoja: si una práctica cultural está viva, no requiere preservación y si está moribunda poco ayuda la preservación a su supervivencia. En ello reside una de las mayores dificultades que enfrenta esta política. Si bien se orienta a valorizar expresiones que en otros contextos y momentos se han entendido como caducas y atrasadas, no considera su régimen de construcción histórica. En otras palabras, ignora los presentes y las relaciones complejas que involucran su transformación constante, la que puede orientarse hacia su desaparición, reinvención o puesta en escena en contextos radicalmente diferentes.

Otro inconveniente de este proceso, que en conjunto denominamos de patrimonialización, tiene que ver con su marco jurídico y la manera como participan quienes lo promueven: las instituciones que rigen las políticas culturales, los intermediarios y las comunidades que se plantean como sus beneficiarias. De esta manera, por ejemplo, las comunidades pueden postular prácticas, bienes y saberes susceptibles de patrimonialización. 
Sin embargo, sus demandas sólo se materializan cuando atienden a los criterios esencialistas de una diferencia cultural domesticada, dictadas por las mismas normas de la Unesco y el Ministerio de Cultura. Así, para que una "manifestación" entre a ser considerada parte de los listados representativos del patrimonio cultural inmaterial, PCI, de la nación, de un departamento o de un distrito, debe ser ratificada por los mandatarios de cada uno de estos órdenes de acuerdo con los dictámenes del Ministerio de Cultura y, recientemente también, del Icanh. A partir de allí se elaboran los planes especiales de salvaguardia, medida final que orienta esta política, la que, como lo detallaremos más adelante, tiene implicaciones financieras regidas por una jerarquización espacial particular.

Una de las dimensiones menos exploradas de este proceso es la relación entre la preservación de las diversas manifestaciones con los intereses de la industria del turismo y la economía cultural que lo acompaña, y la pregunta por cómo incitan a la valoración monetaria de las mismas y a la instrumentalización económica de las identidades de sus productores. En este artículo, avanzaremos sobre estos asuntos con especial atención al proceso de sanción patrimonial que se despliega en Colombia. Examinaremos de manera breve los retos que confronta la construcción de una nación multicultural en torno a los estudios del patrimonio, con el objetivo de reflexionar sobre cómo hacer posible sus usos sociales y no sólo hegemónicos. Propondremos, finalmente, cinco ejes conceptuales que invitan al debate y a la ampliación de la investigación académica sobre el mercado, el consumo y la patrimonialización cultural en Colombia.

\section{DESAFíOS AL ESTUDIO DEL PATRIMONIO CULTURAL EN COLOMBIA}

os estudios sobre el patrimonio en Colombia se han concentrado en el ámbito del patrimonio cultural construido y el Larqueológico. En general, se han desarrollado a partir de las instituciones responsables de su control y administración en los planos regional y nacional (Groot, A.M., 2006; Therrien, M., s.f.). En este sentido, han tenido un radio de acción casi exclusivamente administrativo y sus reflexiones se han orientado hacia 
la valoración, la conservación y la gestión de monumentos, edificaciones y vestigios arqueológicos prehispánicos, articulados a proyectos culturales nacionales y regionales dirigidos desde el Estado. En años recientes, estos trabajos han buscado armonizar los planes de ordenamiento territorial con la salvaguardia de los patrimonios urbanos y arqueológicos. Han alimentado la definición de políticas públicas y la generación de un significativo marco normativo, el más reciente de los cuales incluye el patrimonio intangible o inmaterial, como ya lo hemos indicado (Castellanos, G., 2002; Ministerio de Cultura, 2009b; Therrien, M., 2006; Van der Hammen, M. C., et al., 2009). Sin embargo, no es fácil identificar una línea de acción estatal sostenida frente a la conceptualización, la conservación y la configuración social de los diferentes acervos, colecciones y bienes culturales, tangibles o intangibles; tampoco estrategias de coordinación entre las diferentes entidades encargadas de la gestión del patrimonio cultural (Guerra, W., 200I; Therrien, M., 20IO).

A pesar de que la producción académica ha avanzado en la identificación de estos problemas, todavía hace falta reflexionar sobre el impacto de los marcos ideológicos y los discursos que han predominado en las políticas del patrimonio, como sí se ha hecho en otros países (M. F. Lima Filho y R. M. do Rego Monteiro de Abreu en este número). Es decir, urge indagar sobre si los desarrollos de la patrimonialización, en particular los más recientes, que parecen estar en sintonía con el nuevo proyecto de nación incluyente y multicultural, son en realidad efectivos en la generación de inclusión social y horizontes políticos plurales. Así, por ejemplo, en San Basilio de Palenque, la categoría de patrimonio inmaterial acentúa, en lugar de superar, los sesgos discriminatorios hacia las minorías, en este caso, afrodescendientes, mediante la paradójica inclusión de sus manifestaciones culturales en un plan especial de salvaguardia que de forma velada busca preservar la marginalidad que la declaratoria y su supuesta expansión de derechos intentan superar (Observatorio MIA, 2009; Meza, C. A., 2009; Santoyo, Á., 2009).También habría que examinar las respuestas de los agentes sociales, tanto los incluidos como los excluidos de estas políticas, en un campo de tensiones y disputas por la interpretación de lo que se entiende por patrimonio. Aunque no lo desarrollaremos en este texto, resulta pertinente retornar sobre el campo étnico, ahora que la política étnica se empieza a orientar según la "salvaguarda ante 
el conflicto armado y el desplazamiento forzado" (Corte Constitucional, 2009a, 2009b; Chaves, M., 2010).

De otro lado, si bien las políticas de patrimonio no se presentan directamente como respuesta a los intereses económicos de sus beneficiarios, sí están fuertemente asociadas con preocupaciones sobre la definición de la propiedad, el uso y la circulación de los bienes y saberes patrimonializados (Ministerio de Cultura, 2009b, Decreto 294I). Aquí surge la pregunta sobre el problema de la proyección de los bienes culturales patrimonializables en las industrias culturales y su "sostenibilidad financiera" como bienes protegidos.

Desde el punto de vista de las relaciones entre las declaratorias de patrimonio y el mercado, llama la atención la coincidencia entre la formulación de políticas que propenden por la defensa y preservación de prácticas, saberes e identidades de comunidades locales con la creciente demanda de bienes culturales. En repetidas ocasiones, las políticas de patrimonio han propiciado la mercantilización de los bienes culturales así designados (ver $\mathrm{M}$. Montenegro en este número). El caso del sombrero vueltiao y su sanción legal como símbolo nacional, con la consecuente ampliación de su uso y, por tanto, de su mercado, es tal vez un ejemplo emblemático (Larraín, A., 2009), pero no es el único. En otras ocasiones, la búsqueda de oportunidades económicas ha promovido las acciones de agentes locales en pos de la designación patrimonial; las cuadrillas de San Martín, Meta, (Díaz, I., 20I0; Universidad Nacional e Instituto Departamental de Cultura del Meta, 2009) y el Carnaval de Negros y Blancos (Tobar, J., 2009) son dos ejemplos en este sentido. De tal manera, la multiplicación de instancias que tienen que ver con la ampliación del mercado y las expectativas de los agentes sociales invita al examen de las relaciones entre el consumo, la gestión cultural y la sanción patrimonial. A continuación, nos detendremos en algunas dimensiones de esos asuntos.

En primer lugar, encontramos que agentes que en apariencia siguen lógicas similares en la valoración de bienes culturales como patrimonio, pueden diferir radicalmente en los fines que persiguen y en los modos como participan en el mercado de acuerdo con sus posiciones sociales e intereses económicos y políticos. Como lo muestra el caso del Carnaval de Barranquilla, en los procesos de patrimonialización se generan espacios de 
disputa económica, política y simbólica entre agentes privados, estatales y los grupos sociales involucrados (De Oro, C., 20IO, 2009). Estas controversias giran alrededor de quién decide qué se patrimonializa, cómo se distribuirán los beneficios y quiénes tienen el derecho al uso, la propiedad, la circulación y la distribución de los bienes y saberes patrimonializados.

En este contexto, conviene detenerse en el análisis de saberes y bienes locales y étnicos que hasta hace unos años no eran considerados patrimonio y que hoy son objeto de las políticas y las discusiones sobre el patrimonio y los derechos de propiedad (Greene, S., 2006). Las gastronomías regionales y locales y los debates en torno a los saberes tradicionales de la llamada "cocina fusión” ilustran también esta perspectiva. Mientras se plantea que la sanción patrimonial de saberes y prácticas gastronómicas los valorizan, quienes perciben los mayores beneficios no son sus creadores sino quienes tienen la capacidad de transformarlos y ponerlos a circular en otros circuitos económicos y simbólicos (Arocha, J., 2007; Delgado, R., 2006, 2003). Estos casos pueden contrastarse con otros que llevan los términos de la ecuación a sus límites, aquellos en los que una práctica "tradicional" como el consumo de la hoja de coca o las sesiones de yajé, ponen a prueba la lógica patrimonializante (ver A. Caicedo en este número). El examen cruzado de estas instancias arroja luces sobre el entramado de intereses políticos que subyacen a la idea de que todos los bienes, técnicas y expresiones culturales son, en principio, patrimonializables cuando, según el contexto y las definiciones de legalidad y legitimidad, no todos lo son. Esto nos conduce a considerar el papel de los marcos jurídicos en estos procesos y su implicación en la generación de la desigualdad y la diferencia (Coombe, R. M., 2009; Merry, S. E., 200I).

Precisamente, en el escenario de la patrimonialización sobresale el carácter restrictivo de la legislación y la ambigüedad de la conceptualización que permite definir las expresiones y los productos culturales que pueden ser su objeto. Antes mencionamos los problemas que plantea el carácter restrictivo de la norma pues marca la diferencia entre bienes que pueden o no ser patrimonializados con base en representaciones esencialistas de la cultura y según los marcos legales y políticos que definen esos bienes. Al tiempo, encontramos vaguedad en la definición misma de lo que se entiende por la cualidad inmaterial del patrimonio cultural ya que todas las expresiones 
culturales la tienen pero, al mismo tiempo, ninguna carece de un componente material.

Por otra parte, la valoración del patrimonio inmaterial no es inmune a la jerarquización de escalas. La direccionalidad desde el centro a la periferia en que se toman las decisiones frente a la definición del patrimonio genera asimetrías. Las declaratorias de mayor rango son las de patrimonio de la humanidad de la Unesco, seguidas en Colombia por la lista representativa del PCI que selecciona los ítems que compondrían el acervo de la nación y llegan a recaudar ingresos fiscales (García Canclini, N., I999; Lacarrieu, M., 2008). Los otros listados se reservan para diferenciar e identificar a las regiones y a las localidades y están sujetos a los presupuestos y políticas gubernamentales locales. Así, el estado central, en particular mediante la reglamentación de la Ley II85 de 2008 sobre el patrimonio cultural, ha ordenado que las secretarías de cultura, departamentales y municipales, participen en la creación de un sistema de regionalización cultural y desde antes ha alentado la realización de proyectos departamentales de inventarios de patrimonio intangible coordinados por el Ministerio de Cultura.

Además de la particular articulación de escalas en la normatividad sobre el patrimonio cultural, también vale la pena detenerse en su relación con la economía de mercado. De manera paradójica, las normas internacionales y colombianas proclaman que una dimensión clave de las estrategias de patrimonialización es la salvaguardia y la protección contra la mercantilización (Ministerio de Cultura, 2009b, Decreto 763). No sólo al poner en marcha la salvaguardia se promueve la fijación de las expresiones culturales intervenidas. Al mismo tiempo, las normativas buscan que los entes locales de gobierno procuren los medios para vender los bienes y las técnicas inventariados mediante discursos que promueven el emprendimiento cultural y alientan la transformación de fiestas y carnavales urbanos en espectáculos orientados al turismo y al fomento de las industrias culturales (Castro, S., 2009; J. D. Sanín en este número).

Estudios recientes han comenzado a analizar el impacto que la participación de agentes, gestores culturales y empresas privadas con estrategias comerciales disímiles tiene en los procesos de definición del patrimonio inmaterial (Meza, C. A., 20Io; Santoyo, Á., 2006). La orientación del turismo, impulsado 
como una alternativa de desarrollo importante por las últimas administraciones en Colombia, es equívoca cuando se trata de sopesar el patrimonio cultural frente a las ganancias de esta industria. Así, la Ley II85 de 2008, propone la posibilidad de manejo de aquellos bienes de interés cultural propiedad del Estado, por medio de comodato a agentes privados. Cabe pensar aquí en la coincidencia entre esta orientación con las concesiones ecoturísticas sobre los parques nacionales a empresas privadas. Allí se presume que estas cuentan con mayor capacidad para garantizar la sostenibilidad ambiental y financiera por encima de los habitantes de las localidades vecinas. Sobresale que muchas veces estos pobladores son indígenas, quienes a la vez constituyen uno de los atractivos más importantes en la oferta turística de los parques y reservas naturales, como se puede apreciar en los folletos que promueven el turismo hacia la Amazonia, que prometen entre otros alicientes la "inmersión real y directa en el medio natural y cultural autóctono". Es así como el reciente giro hacia la noción y la definición jurídica del PCI añade un nuevo conflicto sobre la localización, el uso y la circulación de los bienes tradicionales y étnicos que sanciona.

Retornando a los problemas en la conceptualización que sustentan la política del PCI, podemos añadir que al hacerlos aparecer como indisociablemente ligados a una localidad, una esencia y una identidad, se invisibiliza, si no se niega, su constante transformación. Este aspecto controversial, sin embargo, es precisamente el que le permite a los bienes culturales hacer parte de los listados del PCI. A su vez, cifra el valor diferencial de tales bienes en la esfera del consumo (M. Montenegro en este número), promueve las visitas turísticas, hace viable la circulación de bienes y souvenires fuera del lugar de su producción y asocia a los productores con una localidad como la denominación de origen lo hace con los productos de mercado (J. D. Sanín en este número).

Así, la pregunta por el mercado y el consumo cultural en relación con la problemática valoración de lo diverso, muchas veces ignorada, adquiere un lugar central en la aproximación a los procesos de patrimonialización: desnuda su particular uso de la diversidad. Por ejemplo, revela la manera cómo las acciones de una serie de instancias políticas, de especialistas e intermediarios transforman las especificidades de quienes producen los bienes culturales en una abstracción que las integra al patrimonio de la 
humanidad, la nación o la región, entidades a las que empiezan a pertenecer los bienes culturales específicos (KirshenblattGimblett, B., 2007). Desvela, ante todo, las tensiones que afloran de la conceptualización de la diversidad cultural que anima estos procesos, ligada al multiculturalismo celebratorio de la diversidad. Como lo hemos señalado en otro trabajo, este opaca la diversidad mediante un mecanismo ideológico que la despoja de la dialéctica que le confiere historicidad y movilidad (Chaves, $\mathrm{M}$. y Zambrano, M., 2006). Allí reside precisamente la distinción que H. Bhabha (1994) establece entre diversidad cultural y diferencia, donde la segunda aparece como una demanda de singularidad y autonomía. En cambio, en las normativas sobre PCI en Colombia se propone de manera recurrente la representación esencialista de etnicidades, conforme a una política global de identidades afín al neoliberalismo que fija la diferencia en el espacio y niega la movilidad y la densidad histórica que la constituyen (Comaroff, J. y Comaroff, J., 200I, 2009; Segato, R., 2007).

Poner en discusión la incidencia del mercado en la producción del patrimonio, su gestión y su legitimación, para vislumbrar alternativas que amplíen la reflexividad en la formulación de normativas y políticas culturales es un reto que debemos abocar desde el campo académico para potenciar la capacidad de acción y negociación de quienes participan en las diferentes instancias de la política y la industria del patrimonio cultural. En síntesis, nuestras consideraciones tienen en cuenta la normatividad, la articulación de escalas, el sentido de la localización y los usos de diversidad en los procesos de definición del patrimonio. Apuntan también a la necesidad de construir modelos analíticos transdisciplinares que permitan abordar escenarios disímiles y diversos agenciamientos, como los que analizan los autores y autoras reunidos en esta revista.

En esta dirección, los desarrollos teóricos alrededor del patrimonio como instrumento de agenciamiento político podrán encontrarse con aquellos que sustentan la gestión económica de los procesos de patrimonialización, como en el caso del turismo cultural en una localidad indígena del Amazonas, de las noches de yajé (Taussig, M., 1987) convertidas en medicina indígena tradicional en contextos urbanos (A. Caicedo en este número), o de las vicisitudes del sombrero vueltiao de los artesanos sinuanos de Sucre y Córdoba (Larraín, A., 2009). Proponemos así, a continuación, cinco ejes transversales que a nuestro parecer 
permiten reflexionar e investigar sobre las intersecciones entre economía, política y cultura, alrededor de los procesos de patrimonialización y los usos del patrimonio: I. Políticas del patrimonio cultural, 2. Economía política y multiculturalismo, 3. Economía cultural, 4. Identidades, representaciones, diversidad y diferencia, 5. Prácticas de mediación y agenciamiento.

\section{Políticas del patrimonio cultural}

- $\mathrm{n}$ este campo se ponen en juego muchos aspectos clave so- bre las definiciones y los usos del patrimonio y, en especial, - sobre los límites y las posibilidades de los procesos de patrimonialización. En primer lugar, las discusiones sobre lo que debe entenderse por "patrimonio", por "patrimonio cultural", por "patrimonio inmaterial” (M. F. Lima Filho y R. M. do Rego Monteiro de Abreu en este número; Hernández, F., 2002), que derivan inevitablemente hacia la abstracción (o naufragan y se transforman en cuestiones semánticas), evidencian, sin embargo, una serie de intereses en conflicto entre los agentes asociados a estos procesos de denominación. Este trabajo continuo de redefinición del patrimonio supone maneras de comprender la diversidad cultural, las construcciones identitarias y los usos sociales de las expresiones culturales (Kirshenblatt-Gimblett, B., 2004).

Luego, la definición de políticas y estrategias de registro, catalogación, conservación y proyección del patrimonio, entre otras, implica la movilización de redes sociales interesadas en conservar o conseguir beneficios en estos procesos, o bien en defender intereses que estas políticas confrontan o simplemente ignoran (W. A. López en este número). Por ejemplo, la jerarquización y la manera como opera en las sanciones patrimoniales que ya hemos señalado.

La expresión "políticas del patrimonio" alude, por lo tanto, a la construcción de marcos legales e institucionales para los objetos patrimoniales y a los usos políticos de esos mismos objetos. Es por esto que la identificación de la legislación vigente sobre patrimonio y procesos de patrimonialización y su lectura crítica, son indispensables para pensar en temas y problemas que cruzan transversalmente el campo de la patrimonialización. 


\section{ECONOMíA POLÍtica Y MULTiCulturalismo}

 ste eje se concentra en las consecuencias que el llamado "giro cultural", que designa la transición de lo social a lo cultural, ha tenido sobre el patrimonio y la patrimonialización. La asunción del patrimonio como un tema central en las agendas públicas obedece a su particular estatuto cultural. El patrimonio aparece como un bien "público" nacional que refuerza la idea de la viabilidad de lo público y de la necesidad de su defensa en el contexto de la nación. Esto es posible en tanto el patrimonio como objeto cultural es entendido como aislado (y se procura aislarlo) de determinaciones políticas o económicas. No obstante, al atender las conexiones entre estas esferas, se evidencia cómo los valores culturales producidos en los procesos de patrimonialización son redituables y capitalizables.

Al giro cultural ha seguido, casi de inmediato, el multicultural. Esto ha significado el desplazamiento de intereses sociales como la representación política hacia la reivindicación de la diversidad; es decir, hacia la esfera de la "identidad" y el reemplazo de las demandas sociales por las demandas culturales (Chaves, M. y Zambrano, M., 2006; Martínez-Novo, C., 2009). En este escenario, el mercado ha jugado un papel decisivo, puesto que se ha ocupado de la gestión de las identidades de un modo evidente, en ocasiones sobrepasando al Estado. La patrimonialización puede entenderse como un intento por oponer una fuerza identitaria "oficial" a este proceso; es decir, por entrar en la disputa por los marcadores culturales (J. D. Sanín en este número) o aliarse y fundirse con intereses privados que son un activo político de primer orden en las políticas y sistemas de financiación que hoy rigen a los museos (W. A. López en este número) y, como ya lo mencionamos, las concesiones de parques nacionales.

\section{ECONOMía Cultural}

conomía y cultura se han entendido comúnmente como cam- pos inconmensurables (Gudeman, S., I986). La primera trataría Lla concreción y la cuantificación; la segunda, la abstracción y la cualificación. Para ilustrar el modo en que operan estas dicotomías basta con revisar la concepción que, desde la economía 
se tiene de las "industrias culturales" como sectores de la producción que se limitan a una serie de bienes concretos, finales (vv.aa., 200I).

Esto demuestra, entre otras cosas, que la categoría "industria cultural" (que en ese giro resulta, paradójicamente, limitada a la producción de formas de la "alta cultura" y de los medios masivos de comunicación) resulta insuficiente para salvar la diferencia entre economía y cultura, en tanto se concentra en la producción, a la vez que se resiste al uso de una definición amplia de "cultura". Por lo tanto, es necesario acercarse a teorías contemporáneas que integran el consumo a las redes sociales y culturales y no lo limitan a un intercambio más o menos objetivo de bienes (Lash, S. y Urry, J., 1998; Ortiz, R., 2004). De allí la utilidad de nociones como "cultura de consumo" (Featherstone, M., 2000) o "economía cultural” (Groys, B., 2005).

La hipótesis de la economía cultural tiene su origen en la idea de usar (traducir) los conceptos básicos de las ciencias económicas para comprender de otro modo los fenómenos culturales: producción, circulación, capital, valor (de uso, de cambio, de signo), plusvalía, interés, etc. (Baudrillard, J., 1974). De allí que nociones como "capital cultural" (Bourdieu, P., 2003) hayan ganado espacio en la teoría y demostrado su utilidad analítica y operativa. En este sentido, la economía cultural designaría más un cuerpo conceptual, un método, que un campo concreto o un objeto de estudio. No se trata, pues, de una economía de la cultura. El concepto de economía cultural resulta muy útil porque engloba categorías como "mercado" y "consumo" y hace posible su articulación con objetos y procesos culturales como el patrimonio (Comaroff, J. y Comaroff, J., 2009; Kirshenblatt-Gimblett, B., 2007). Habría, pues, una "economía del patrimonio" que debe ser reconocida y estudiada, como lo propone M. Montenegro en este número.

\section{IDENTIDADES, REPRESENTACIONES, DIVERSIDAD Y DIFERENCIA}

n los discursos sobre patrimonio material o inmaterial es muy
común el uso de la retórica de la diversidad y la autenticidad
cultural. En ellos la diversidad se concibe como algo dado que 
existe afuera para ser aprehendido, evidente y al mismo tiempo condición propia de la cultura, su determinante (Fraser, N., I997; Friedman, J., 200I; Scott, J., 1993). Niega de este modo que sea producto de asimetrías de poder y regímenes de representación históricos que producen la diferencia que encierra (Derrida, J., I967; Hall, S., 2000). La autenticidad a su vez, ligada a la noción de identidad-identificación se piensa en general como ajena al mercado y al consumo, que desde esta perspectiva la corrompe. Sin embargo, el acto de la identificación como lo propone J. Friedman (200I), supone "el consumo de símbolos que nos definen pero que no producimos sino que obtenemos en el mercado" y por lo tanto es en sí mismo inauténtico o por lo menos ambiguo, ya que al mismo tiempo supone y niega la autenticidad. Esto implica la erosión de la búsqueda de autenticidad del bien que en cambio supone la nostalgia del bien perdido (Gonçalves, J. R. S., 2000; Povinelli, E., 2002).

Identidad, diversidad, diferencia, aparecen aquí como activos económicos; como capitales. De otro lado, la utilidad que estas categorías pudieran tener para el agenciamiento político de los sujetos sociales involucrados en los mercados del patrimonio queda neutralizada en este proceso, como lo sugiere la escena turística descrita por Gallego (2009) entre los tikuna de la comunidad de La Libertad ubicada sobre el río Amazonas en territorio colombiano. Parte del problema estriba en la escisión economía-cultura citada arriba. Así, por ejemplo, las teorías de gestión cultural, interesadas por la capitalización de recursos, se encuentran con el uso político de la retórica de la diversidad cultural como garante del desarrollo sostenible, que comúnmente abstraen las condiciones económicas objetivas de los sujetos o comunidades sobre quienes recae la patrimonialización como en el caso de San Basilio de Palenque (Observatorio MIA, 2009).

\section{Prácticas de mediación y agenciamiento}

$\rho_{\text {ti }}^{o}$ or último, proponemos como un eje analítico clave, la perspectiva de las apropiaciones del patrimonio y de la patrimonialización desde diversos sujetos sociales inmersos en una red de complejas significaciones y negociaciones sobre sus redes sociales y las de sus otros (Bourdieu, P., I977; De Certeau, M., 
I996; Giddens, A., I995; Massey, D., 1994). Esto implica considerar las relaciones de los productores culturales y los funcionarios estatales interesados en este proceso, sin perder de vista el papel de mediación de diversos agentes entre los que se pueden identificar profesionales de las ong, antropólogos y gestores culturales. Todos ellos son sujetos con roles económicos y políticos diferentes que actúan en contextos específicos, que deben ser investigados, y donde el traslape de escalas regionales, nacionales o internacionales, dependiendo de su posición, los afecta de manera disímil. Esta indagación por el entramado de interacciones y negociaciones puede revelar el ámbito económico y político en el que tiene lugar la patrimonialización y ofrecer, a la vez, una manera de identificar los límites que imponen las condiciones sociales y económicas a la capacidad de acción de cada uno de esos agentes dependiendo de su posición. También abre una ventana a la comprensión de la compleja dinámica de las redes sociales locales en interacción con escalas más amplias (Friedman, J., 200I; Hall, S., 2000; Massey, D., I994). Así, por ejemplo, los propósitos y las consecuencias de las políticas estatales de patrimonialización son diferentes según el lugar que ocupa la comunidad postulada en un juego de jerarquías que se rigen de acuerdo con la cartografía socio-racial de la nación, la valoración en la escala de autenticidad y de alteridad, y en la del capital socioeconómico y simbólico.

\section{CONSIDERACIONES FINALES}

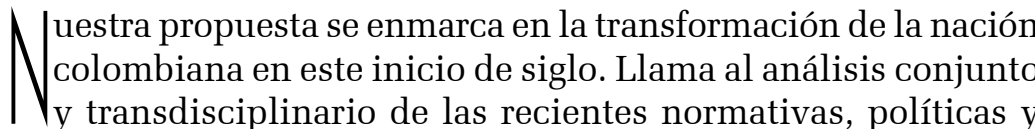
prácticas que han redefinido la noción de interés público alrededor de las expresiones culturales de grupos y colectivos marcados por una supuesta diferencia irreducible. Buscamos ampliar de este modo la discusión académica sobre la patrimonialización y fundamentar una perspectiva más estratégica que tome en cuenta los intereses de los diversos agentes en ella implicados.

Hemos indicado también que sólo mediante el estudio juicioso de diversos escenarios y el análisis comparativo será posible elaborar una interpretación sobre la explosión de identidades étnicas 
y culturales representadas como "tradicionales" y los diversos agenciamientos en los contextos generados por la ejecución de las políticas del PCI y la expansión del consumo y el mercado cultural. Como ya lo han sugerido otros autores, la alusión a la pertenencia innata o al sentimiento primordial por parte de estos colectivos se activa para reclamar derechos de propiedad cultural (Brown, M. F., 2003; Coombe, R. M., I998), a medida que sus conocimientos se convierten en objeto de comercio (Greene, S., 2006), su espiritualidad en experiencia de consumo (Sarrazin, J. P., 2006), sus fiestas populares en eventos espectaculares (Yomaiel, C., Lacarrieu, M. y Maronese, L., 2008), y sus expresiones en mercancías para las urbes y el turismo (Comaroff, J. y Comaroff, J., 2009).

Queremos cerrar con la insistencia en tejer estas aproximaciones académicas, y las propuestas de los siguientes artículos, con la preocupación sobre las políticas públicas culturales y la transferencia de conocimientos a los grupos de base que participan en la industria del patrimonio.

\section{RefEREnCIAS}

Arocha, J. (2007). Encocaos con papa, iotro etnoboom usurpador? Revista Colombiana de Antropología, 43, 9I-II7.

Baudrillard, J. (I974). Crítica de la economía política del signo. México: Siglo XXI.

Bнавна, H. (1994). El lugar de la cultura. Buenos Aires: Manantial.

Bourdieu, P. (1977). Outline of a Theory of Practice (Richard Nice, Trad.). Cambridge: Cambridge University Press. . (2003). Creencia artística y bienes simbólicos. Elementos para una sociología de la cultura. Buenos Aires: Aurelia Rivera.

Brown, M. F. (2003). Who owns native culture? Londres: Harvard University Press.

Camarero Izquierdo, C. y Garrido Samaniego, M. J. (2004). Marketing del patrimonio cultural. Madrid: Pirámide.

Castellanos, G. (2002). Régimen jurídico del patrimonio arqueológico en Colombia. Bogotá: ICANH. 
Castro, S. (2009). Las políticas culturales como un patrimonio de la nación. En Ministerio de Cultura. Compendio de políticas culturales (Documento de discusión 2009) (pp. 493-496). Bogotá: autor.

Chaves, M. (Ed.) (2010). La multiculturalidad estatalizada. Indígenas, afrodescendientes y configuraciones de estado en Colombia. Bogotá: ICANH, inédito.

Chaves, M. \& Zambrano, M. (2006). From blanqueamiento to reindigenización: paradoxes of mestizaje and multiculturalism in contemporary Colombia. European Review of Latin American and Caribbean Studies/ Revista Europea de Estudios Latinoamericanos y del Caribe, 80, 5-23.

Colombia, Corte Constitucional (2009a). Auto $\mathrm{N}^{\circ} 004$ de 2009, Protección de los derechos fundamentales de las personas y los pueblos indígenas desplazados por el conflicto armado o en riesgo de desplazamiento forzado. Bogotá: Autor.

. (2009b). Auto $\mathrm{N}^{\circ}$ o05 de 2009, Protección de los derechos fundamentales de la población afrodescendiente víctima del desplazamiento forzado. Bogotá: Autor.

Colombia, Ministerio de Cultura. (200I). Plan Nacional de Cultura 200I2210. Hacia una ciudadanía democrática cultural: un plan colectivo desde y para un país plural. Bogotá: Autor.

. (2009). Compendio de políticas culturales. Documento de discusión 2009. Bogotá: Autor.

Colombia, Ministerio de Cultura, Dirección de Patrimonio, Grupo de Patrimonio Inmaterial. (1997). Ley general de cultura-Patrimonio arqueológico. Bogotá: ICANH.

- (200I). Vigía del patrimonio. Bogotá: Autor.

. (2007). Manual para la implementación del proceso de identificación y recomendaciones de salvaguardia de las expresiones del patrimonio cultural inmaterial. Bogotá: ICANH-Ministerio de Cultura.

. (2009). Normas generales para la gestión, protección y salvaguardia del patrimonio cultural en Colombia. Bogotá: Autor.

Colombia, Observatorio de Patrimonio Cultural MiA. (2008). Evaluación de las políticas de patrimonio inmaterial [Base de datos]. Recuperado de: http://erigaie.org/index.html?modulo=observatorio\&artic ulo_id $=4$

. (2009). Percepciones sobre el proceso de declaratoria en San Basilio de Palenque. Recuperado de: http://erigaie.org/index.ht $\mathrm{ml}$ modulo $=$ observatorio\&articulo_id $=4$ 
Condominas, G. (2004). Investigación y salvaguardia del patrimonio inmaterial. Museum International, LVI(I-2/22I-222), 2I-3I.

Coombe, R. M. (2009). The expanding purview of cultural properties and their politics. Annual Review of Law and Sociology, 5, 393-4I2.

Comaroff, J. \& Comaroff, J. (200I). Millenial capitalism: First thoughts on a second coming. En J. Comaroff \& J. Comaroff (Eds.). Millenial capitalism and the culture of neoliberalism (pp. I-56). Durham: Duke University Press.

Comaroff, J. \& ComarofF, J. (2009). Ethnicity Inc. Chicago: The University of Chicago Press.

De Certeau, M. (I996). La invención de lo cotidiano (Vol. I). México: Universidad Iberoamericana.

De Oro, C. (2009). Tradición y modernidad: el carnaval de Barranquilla y la pugna entre lo cultural, lo económico y lo político. En: M. S. Lizcano \& D. González Cueto (Comps.). Leyendo el carnaval: miradas desde Barranquilla, Bahía y Barcelona (pp. 37-58). Barranquilla: Ediciones Uninorte.

. (en prensa). Las paradojas de la preservación de las tradiciones del carnaval de Barranquilla en medio del mercantilismo, la globalización y el desarrollo cultural. Revista Brasileira do Caribe.

Delgado, R. (2003). Diversidad de sabores, olores, texturas, sonidos, imágenes, memorias y relato de las comidas y medicinas en San Basilio de Palenque. En: Etnografías y patrimonios. Relatos de San Basilio de Palenque (Tomo 3) [CD]. Medellín: Departamento de Antropología de la Universidad de Antioquia.

. (2006). De mi mamá al laboratorio de comidas y culturas. En VII Encuentro para la promoción y difusión del patrimonio inmaterial de países iberoamericanos. Gestión del patrimonio inmaterial y la diversidad cultural-Memorias. (pp. 469-482). Bogotá: Autor.

DíAz, I. (20IO). Patrimonialización, construcción de identidades y formación del estado en Puerto Santander y San Martín, Meta (Trabajo de grado sin publicar). Carrera de Antropología, Pontifica Universidad Javeriana, Bogotá.

FeAtherstone, M. (2000). Cultura de consumo y posmodernismo. Buenos Aires: Amorrortu.

FRASER, N. (1997). Iusticia Interrupta. Reflexiones críticas desde la posición postsocialista. Bogotá: Siglo del Hombre-Uniandes.

Friedman, J. (200I). Identidad cultural y proceso global. Buenos Aires: Amorrortu. 
García Canclini, N. (I999). El consumo cultural: una propuesta teórica. En G. Sunkel (Coord.). El consumo cultural en América Latina (pp. 26-49). Bogotá: Convenio Andrés Bello.

GiDDEns, A. (I995). Modernidad e identidad del yo: El yo y la sociedad en la época contemporánea. Barcelona: Gedisa.

GonÇALves, J. R. S. (I996). A retórica da perda. Os discursos do patrimônio cultural no Brasil. Rio de Janeiro: UFRJ-IPHAN.

GreEne, S. (2006). ¿Pueblos indígenas S.A.? La cultura como política y propiedad en la bioprospección farmacéutica. Revista Colombiana de Antropología, 42, I79-22I.

Groot, A. M. (2006). Arqueología y patrimonio: conocimiento y apropiación social. Revista de la Academia Colombiana de Ciencias, 3O(II4): 5-I7.

Groys, B. (2005). Sobre lo nuevo. Ensayo de una economía cultural. Valencia: Pre-Textos.

Gudeman, S. (I986). Economics as culture: models and metaphors of livelihood. Londres: Routledge.

. (200I). The anthropology of economy: Community, market, and culture. Londres: Malden-Blackwell.

GuERRA, W. (200I). Del exotismo a la autonomía. Los pueblos indígenas y los cambios en los conceptos de identidad, patrimonio y museo. En La arqueología, la etnografía, la historia y el arte en el museo. Memorias de los coloquios nacionales (pp.I63-I70). Bogotá: Ministerio de Cultura-Museo Nacional de Colombia.

Hall, S. (2000). Identidad cultural y diáspora. En S. Castro, O. Guardiola \& C. Millán (Eds.). Pensar (en) los intersticios. Teoría y práctica de la crítica poscolonial (pp.I3I-I45). Bogotá: Pontificia Universidad Javeriana e Instituto Pensar.

. (I997). El trabajo de la representación. En Stuart Hall (Ed.),

Representation: cultural representations and signifying practices (pp. I3-74). Londres: Sage.

HeRnÁnDEZ, F. (2002). El patrimonio cultural: La memoria recuperada. Gijón: Trea.

Kirshenblatt-Gimblett, B. (2004). El patrimonio inmaterial como producción metacultural. En Unesco (Ed.), Museum International. Patrimonio Inmaterial (p.p. 52-67 ). (Vols. 22I-222). Paris: Unesco.

. (2007). World heritage as cultural economics. En: I. Karp et al. Museum frictions. Public culture/Global transformations (pp.I6I202). Durham: Duke University Press. 
LACARRIEU, M. (2008). ¿Es necesario gestionar el patrimonio inmaterial? Notas y reflexiones para repensar las estrategias políticas y de gestión. Boletín Gestión Cultural, I7, I-26 [Número especial: Gestión del patrimonio inmaterial].

LARRAín, A. (2009). A patrimonialização da arte e da cultura indígena na Colômbia. O caso do sombrero vueltiao. Revista Tellus, I7, 207229.

Lash, S. y URrY, J. (1998). Economías de signos y espacios. Buenos Aires: Amorrortu.

Massey, D. (1994). Space, place and gender. Minneapolis: University of Minnesota Press.

Merry, S. E. (200I). Changing rights, changing culture. En: J. Cowan et al. (Eds.), Culture and Rights. Anthropological perspectives (pp.3I-55). Cambridge: Cambridge University Press.

Meza, C. A. (2009). Comentarios al PES de Palenque de San Basilio. Manuscrito sin publicar, ICANH, Bogotá.

. (20IO). Patrimonialización cultural y desarrollo regional. La situación de los pueblos afrochocoanos en la vía al mar. En M. Chaves (Ed.). La multiculturalidad estatalizada. Indígenas, afrodescendientes y configuraciones de estado en Colombia y Latinoamérica (pp.239-249). Bogotá: ICANH, inédito.

Ortiz, R. (2004). Mundialización y cultura. Bogotá: Convenio Andrés Bello.

PovinelLi, E. (2002). The cunning of recognition: Indigenous alterities and the making of Australian multiculturalism. Durham: Duke University Press.

Rosas Mantecón, A. (2005). Las disputas del patrimonio. En: N.García Canclini (Coord.) La antropología urbana en México (pp. 60-95). México: Fondo de Cultura Económica-Conaculta-UNAM.

SANTOYO, Á. (2006). Investigación para la definición de un marco conceptual para la política de PCI en Colombia. Recuperado de: http:// erigaie.org/index.html?modulo=observatorio\&articulo_id $=4$

. (2009). Comentarios generales sobre el borrador del Plan Especial de Salvaguardia (PES) Palenque de San Basilio. Manuscrito sin publicar. ICANH, Bogotá.

SARRAzIN, J. P. (2006). Idées globalisées et constructions locales. L'image valorisée del'indianité dan la Colombie contemporaine. Autrepart, $38,155-172$.

Scotт, J. (I993). Igualdad versus diferencia: los usos de la teoría postestructuralista. Debate Feminista, 4(8), 85-104. 
Segato, R. (2007). La nación y sus otros: raza, etnicidad y diversidad religiosa en tiempos de política de la identidad (pp. 37-69). Buenos Aires: Prometeo libros.

THERRIEN, M. (s. f.). Preservación del patrimonio cultural nacional. Bogotá: ICANH-Colcultura.

. (2006). Comentarios al documento: valores y criterios de valoración para declaratoria de bienes materiales como BIC. Recuperado de: http://erigaie.org/index.html?modulo=observatori o\&articulo_id $=4$.

. (20I0). "Los dilemas de las políticas culturales de patrimonialización en Colombia”. En Margarita Chaves (Ed.). La multiculturalidad estatalizada. Indígenas, afrodescendientes y configuraciones de estado en Colombia y Latinoamérica (223-237). Bogotá: ICANH, inédito.

TAussig, M. (I987). Chamanismo, colonialismo y el hombre salvaje. Un estudio sobre el terror y la curación. Bogotá: Editorial Norma.

ToBar, J. (Director). (2009). Carros alegóricos. Carnaval de Pasto [DVD]. Colombia: Universidad Nacional de Colombia, Ieco, Quinde Audiovisuales, Universidad del Cauca, Gicea, Fondo Mixto de Cultura de Nariño.

UnesCo. (2003). Convención para la salvaguardia del patrimonio cultural inmaterial. Recuperado de: http://www.portal.unesco.org/es/

Universidad Nacional de Colombia \& Instituto Departamental de Cultura DEL META (2009). Expedición de patrimonio material e inmaterial en el Departamento de Meta. Manuscrito si publicar.

VAn der Hammen, M. C., Lulle, T \& Palacio, D. C. (2009). La construcción del patrimonio como lugar: Un estudio de caso en Bogotá. Antípoda, $8,6 \mathrm{I}-85$.

VV. AA. (200I). Economía y cultura: la tercera cara de la moneda. Memorias del seminario. Bogotá: Convenio Andrés Bello.

Yomaiel, C., LaCARRieu, M. \& Maronese, L. (2008). Carnaval porteño: entre la fiesta y el espectáculo. Manuscrito en preparación.. 